\title{
The Prediction Model of Influence of Small Radius UTLT Curvature on Fire Site Visibility Based on Pyrosim Multiple Regression Analysis
}

\author{
Xiao Ju Li ${ }^{1 *}$, Kun $\mathrm{Yao}^{1}$, Jun Yu Dai ${ }^{2}$, Yun Long Song ${ }^{3}$ \\ ${ }^{1}$ College of Resource and Environment, Xichang University, Xichang 615013, Sichuan, China \\ ${ }^{2}$ Sichuan Academy of Safety Science and Technology, ChenDu 610045, Sichuan, China \\ ${ }^{3}$ China Academy of Building Research, BeiJing100083, China
}

\begin{abstract}
As a modern city's "fourth dimension space", UTLT is a typical narrow and confined underground space. Due to the special structural position and the curvature of the tunnel, the hot smoke in the fire field can easily form a "smoke turning circle" phenomenon, which greatly reduces environmental visibility and threatens people escape quickly. This article takes a UTLT small radius tunnel as an example to investigate the relationship between the curvature of the tunnel and the visibility of the fire near the fire source. Firstly, using Pyrosim to simulate the change of visibility in different curvatures of fire field, and using SPSS to carry out multiple regression analysis on numerical simulation data, summarizing the prediction model of the impact of small radius UTLT tunnel curvature on the visibility near the fire source. Finally, regression model analysis and test show that the curvature is negatively correlated with the visibility of the tunnel and the prediction model is feasible. The research results can provide theoretical guidance for the design of UTLT-type small-radius circulation smoke evacuation and rapid evacuation of personnel, and provide a reference for similar curve tunnel curvature design and smoke control measures.
\end{abstract}

\section{Introduction}

UTLT is the underground transportation tunnel in urban areas and belongs to a new type of urban underground transportation system. As the typical representative of the "fourth dimension space" of the city, UTLT achieves an efficient transformation of urban underground space in the space-time dimension ${ }^{[1-4]}$. UTLT small-radius tunnels have large curvature and belong to the typical underground narrow-length confined space ${ }^{[5-6]}$. The structure of the tunnel is complex, with features such as small cross-sectional area, closed space, large slope, and multiple entrance and exit ramps. The fire can easily form the phenomenon of "smoke turning" at the curvature of the tunnel ${ }^{[7-9]}$.In addition, due to the low height of UTLT, the flue gas is settled quickly. Once a fire occurs, the visibility of the fire in the tunnel will be seriously affected, which is extremely unfavorable to the escape and rescue of safety evacuation.

At present, there are many studies on tunnel disaster prevention and reduction. As one of the major disasters, tunnel fires have been studied by many scholars at home and abroad. Thomas ${ }^{[10]}$ studied the fire conditions of the tunnel under vertical ventilation conditions, and finally obtained the theoretical equation of the hot flue gas return upstream of the fire source. Kunikane et $\mathrm{a}^{[11]}$. conducted a numerical simulation of the length of the return flow of smoke from a stationary vehicle in a tunnel. Studies have shown that the presence of vehicles in the tunnel makes the return flow length of fire smoke smaller. Hu Longhua analyzed large-scale and full-scale field experiments on the horizontal smoke exhaust method of the tunnel fire, the optimal design of the combined smoke, the timing of the smoke exhaust, and the optimal control of the vertical smoke exhaust method in the early stage of the fire, and provided experimental data support ${ }^{[12]}$. Huang Jianbo $^{[13]}$ analyzed the influencing factors of the visibility of subway tunnel fire through orthogonal test. The results show that the flue gas spreading distance is the most affected by the ventilation wind speed when the visibility is at the critical value. $\mathrm{Xu}$ Hongtao et al ${ }^{[14]}$. used CFD technology to simulate the influence of different production volumes of an underground garage on visibility, and finally concluded that the amount of smoke produced was the key to the design of smoke extraction systems.

In summary, most of the studies on tunnel fires are flue gas simulations of conventional straight tunnel fires, etc. The research on UTLT tunnels focuses on the analysis of the influence of ventilation wind speed, fire source power and other factors on the smoke flow in tunnel fires, There is less research on the radius curvature of the UTLT, and the influence of the curvature on the visibility of the fire field is less common. This paper takes a UTLT small-radius tunnel

* Corresponding author: Xiao Ju Li. E-mail addresses: lixiaojuxcv@163.com 
as an example. Firstly, Pyrosim software was used to simulate the change of visibility in different curvature fire environment. The SPSS software and multiple regression analysis was performed on the simulation data of the curvature of the orbit and the visibility of the fire field. Finally, a prediction model for the influence of the curvature of a small radius UTLT on the visibility of the vicinity of a fire source is proposed. The research results can provide theoretical guidance for smoke prevention design and personnel safety evacuation of UTLT type small-radius tunnels, and provide reference for curved tunnel curvature design and smoke control measures.

\section{UTLT Project Overview}

In this study, taking a small radius UTLT as an example, the main trunk of the tunnel is ring-shaped, with eight main entrances and exits, and the length of the main ring road is about 3 kilometers. The UTLT has more than 40 lanes and three lanes in one direction. The structure affected by the curvature is more complicated. The traffic flow in the ring road is large and belongs to the typical small radius curve tunnel. The UTLT structure diagram and the tunnel curve structure are shown in Figure 1 to Figure 2.

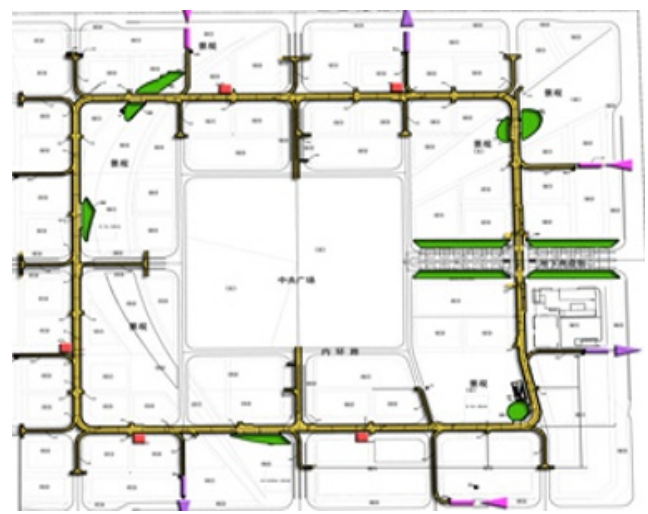

Fig. 1. UTLT structure

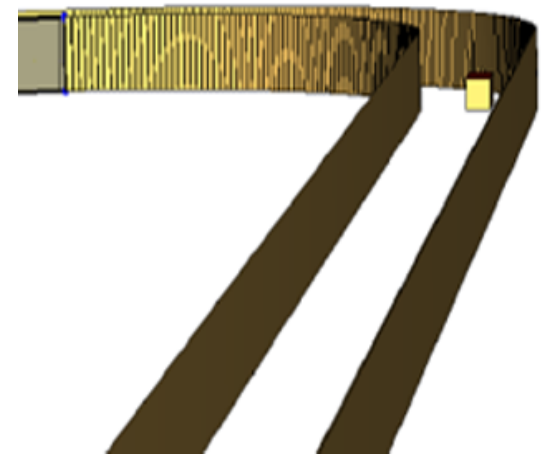

Fig. 2. Curve tunnel

\section{Research methods}

\subsection{PyroSim fire numerical simulation}

PyroSim is a pre-processing and post-processing software dedicated to fire dynamics simulation developed by Thunderhead Engineering Inc. based on $\operatorname{FDS}^{[15-16]}$.

There are many advantages of PyroSim software. It not only accurately simulates changes in fire environment visibility, flue gas temperature, heat release rate of fire source, and $\mathrm{CO}$ concentration, but also visual editing and three-dimensional graphical pre-processing. Compared with FDS, modeling is convenient. Faster and more optimized calculation methods ${ }^{[3,16-18]}$. In this study, we use Pyrosim software to model the curvature of a small radius UTLT and focus on the change of visibility parameters in the fire environment.

\subsection{Multiple Regression}

An equation describing the dependent variable $\mathrm{Y}$ as an independent variable $\chi_{1}, \chi_{2}, \ldots, \chi_{n}$ and an error term

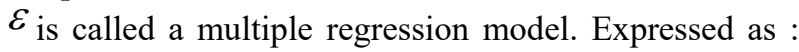
$Y=\beta_{0}+\beta_{1} \chi_{1}+\beta_{2} \chi_{2}+\cdots+\beta_{\mathrm{n}} \chi_{n}+\varepsilon$. In the formula, $\beta_{0}, \beta_{1}, \beta_{2}, \cdots \beta_{\mathrm{n}}$ are regression parameters; $\varepsilon$ is an error term. A linear regression model with explanatory variables is expressed as: $Y_{i}=\beta_{1}+\beta_{2} X_{2 i}+\beta_{3} X_{3 i}+\ldots+\beta_{k} X_{k i}+u_{i}$

The parameters $\beta_{j}(j=1,2, \ldots, k)$ in the model are partial regression coefficients and the sample size is $n$ [19]

In a multiple regression model, the goodness-of-fit test is usually judged by multiple coefficients $R^{2}$. Multiple regression

$$
\begin{aligned}
& \hat{Y}_{i}=\hat{\beta}_{1}+\hat{\beta}_{2} X_{2 i}+\hat{\beta}_{3} X_{3 i}+\ldots+\hat{\beta}_{k} X_{k i} \\
& R^{2}=\frac{\text { ES S }}{\text { TS S }}=\frac{\sum\left(\hat{Y}_{i}-\bar{Y}\right)^{2}}{\sum\left(Y_{i}-\bar{Y}\right)^{2}}=\frac{\text { TS S - R S S }}{\text { TS S }}=1-\frac{\sum e_{i}^{2}}{\sum y_{i}^{2}}
\end{aligned}
$$

The revised coefficient was revised to:

$$
\bar{R}^{2}=1-\frac{R S S / n-k}{T S S / n-1}=1-\frac{n-1}{n-k} \frac{\sum e_{i}^{2}}{\sum y_{i}^{2}}, 0 \leq R^{2} \leq 1,
$$

The $R^{2}$ closer to 1 , the higher the degree of $\mathrm{fit}^{[19]}$.

Hypothesis : $H_{0}: a_{1}=a_{2}=\cdots a_{p}=0$. The sum of the square of the total deviation of the dependent variable $\mathrm{Y}$ from the average is:

$$
\begin{aligned}
& Q_{Y}=\sum_{i=1}^{n}\left(Y_{i}-\bar{Y}\right)^{2}=\sum_{i=1}^{n}\left(Y_{i}-\hat{Y}\right)^{2}+2 \sum_{i=1}^{n}\left(Y_{i}-\hat{Y}\right)\left(Y_{i}-\bar{Y}\right)^{2}+\sum_{i=1}^{n}(\hat{Y}-\bar{Y})^{2} \\
& \text { Because of : } \sum_{i=1}^{n}\left(Y_{i}-\hat{Y}\right)\left(Y_{i}-\bar{Y}\right)^{2}=v^{T} A \beta=0
\end{aligned}
$$

and

$$
\sum_{i=1}^{n}\left(Y_{i}-\hat{Y}\right)\left(Y_{i}-\bar{Y}\right)^{2}=v^{T} A \beta=0
$$

$$
R^{2}=\frac{Q_{R}}{Q_{Y}}, 0 \leq R^{2} \leq 1 \text {, The R2closer to } 1,
$$
the fitting degree is higher. 
$F=\frac{\frac{Q_{R} / p}{\sigma^{2}} / p}{\frac{Q_{\varepsilon} /(n-p-1)}{\sigma^{2}}(n-p)} \sim F(p, n-p$

Through the level of significance, and check the $\mathrm{F}$ distribution table, we can conclude if

$$
F>F(p, n-p-1), \quad H_{0} \text { is rejected. That }
$$
means the regression model has significant effect.

\section{Pyrosim simulation and analysis}

\subsection{Fire model establishment}

This paper uses a small radius UTLT as a simulation object. It is proposed to use Pyrosim fire simulation software to establish the tunnel fire model. Refer to the relevant information and consider the maximum unfavorable principle to simulate the fire source power to take 10MW. According to relevant design specifications, the vertical ventilation wind speed value is $3 \mathrm{~m} / \mathrm{s}$. The fire source position is set at the curvature of the tunnel. See Figure 3, the grid is divided into $0.2 \times 0.2 \times 0.2 \mathrm{~m}$. Visibility in UTLT fires is affected by many factors. This paper mainly analyzes the effect of UTLT curvature on the environmental visibility near the fire source in the cases of curvature ratios of $1 / 10,1 / 20$, and $1 / 30$. The safety margin of the visibility at the clear layer height of $2 \mathrm{~m}$ is $10 \mathrm{~m}$, and the visibility measurement point is set at the position of the UTLT fire source, at a position $30 \mathrm{~m}$ apart from the downwind zone and $2 \mathrm{~m}$ from the bottom of the tunnel. The fire simulation model is shown in Figure 4:

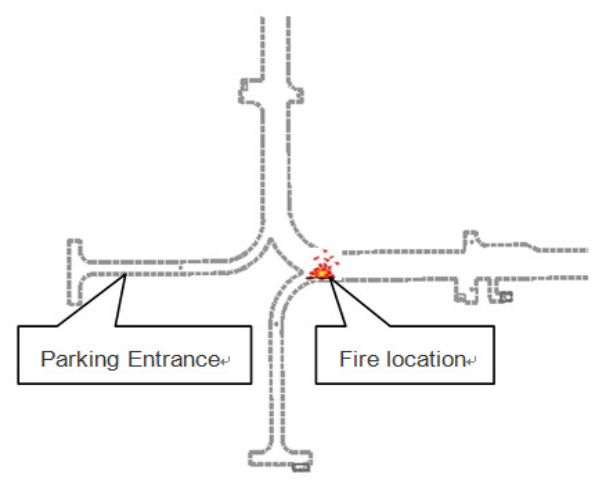

Fig.3. UTLT simulated fire location

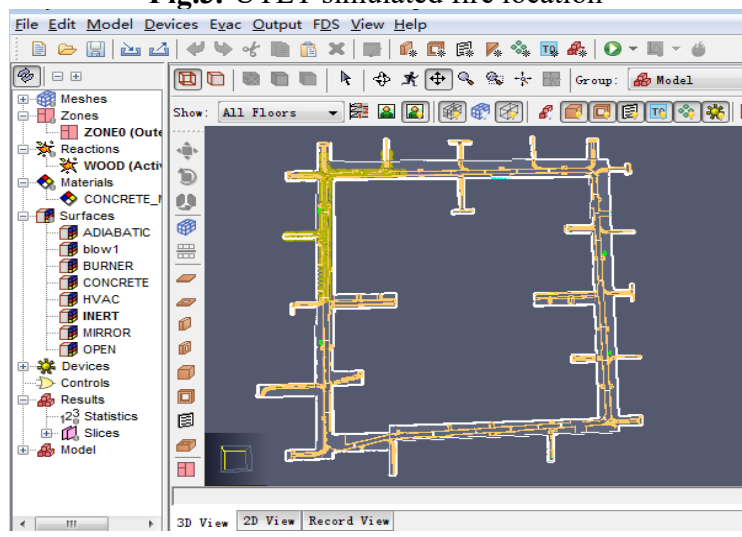

Fig.4 - chematic diagram of the UTLT Pyrosim model

\subsection{UTLT fire visibility analysis}

Analysis of the numerical simulation data can obtain the relationship between the visibility and curvature of the ring road near the fire source location at $30 \mathrm{~m}$ and the location of the fire source, as shown in Figure 5, and the specific changes of the fire site visibility can be seen in Figures 6 to8. It can be seen from the figure that the visibility near the fire source in the ring road decreases with the increase of the fire time. The minimum environmental visibility of the fire site is less than $4 \mathrm{~m}$, which seriously affects the quick escape of the personnel. The greater the UTLT curvature is, the lower the visibility is near the fire source. The visibility of the fire field in the small radius curvature tunnel is much lower than $10 \mathrm{~m}$, which poses a great threat to the rapid escape of personnel. The curvature of the loop is one of the important factors affecting the visibility of the fire.

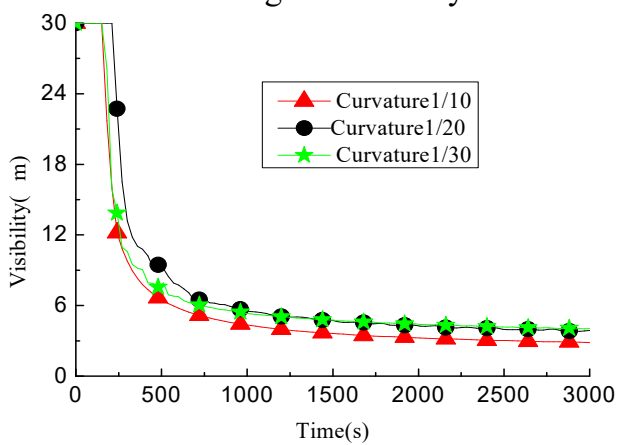

(a) Fire location

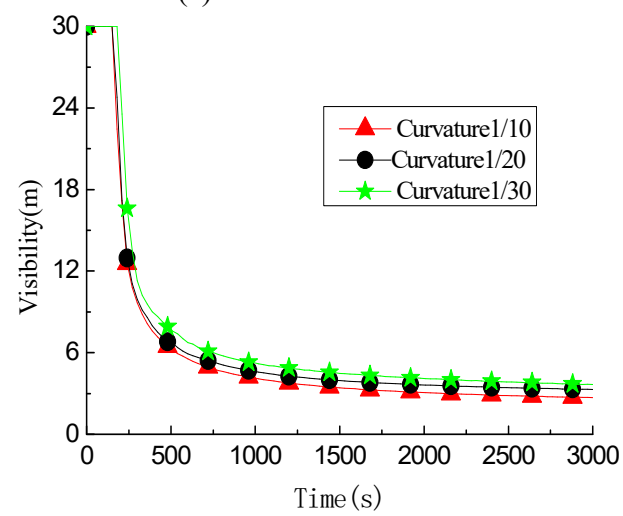

(b) Upwind $10 \mathrm{~m}$

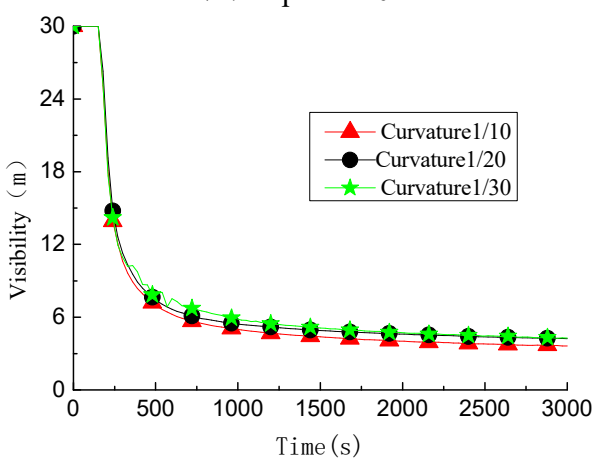

(c) Downwind $10 \mathrm{~m}$ 


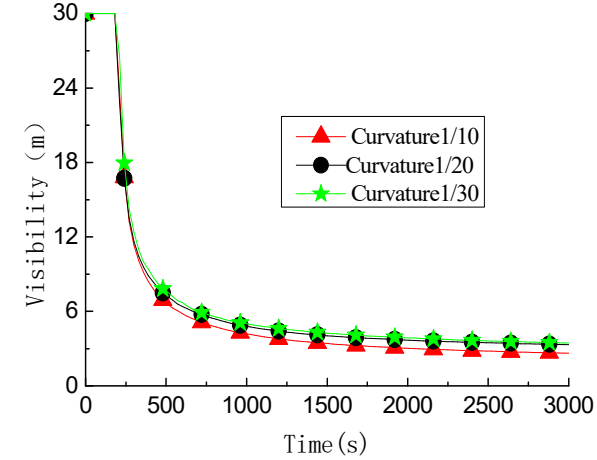

(d) Upwind $30 \mathrm{~m}$

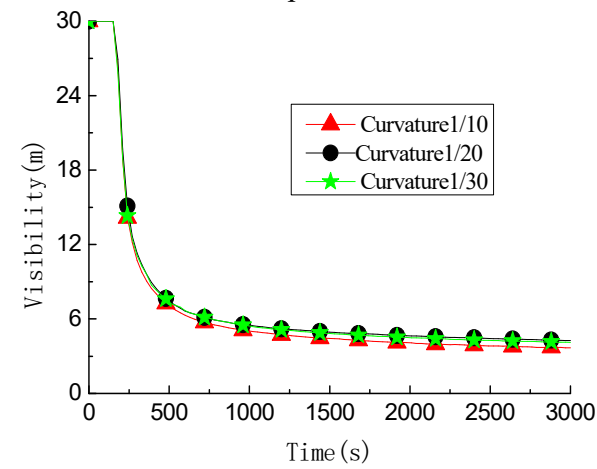

(e) Downwind $30 \mathrm{~m}$

Fig.5. Visibility of UTLT near the fire under different curvatures

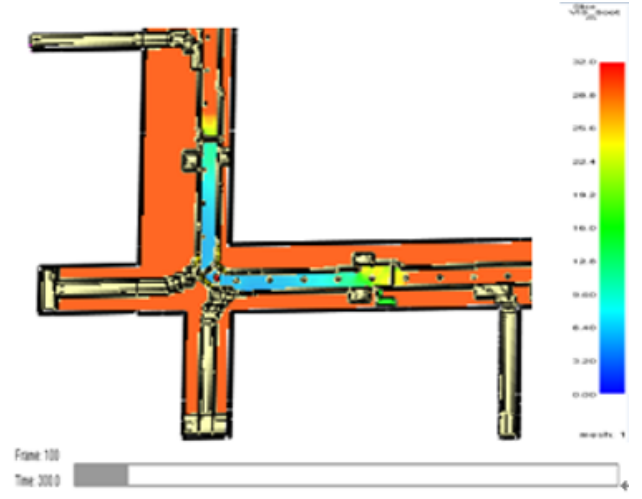

(a) 300s fire visibility

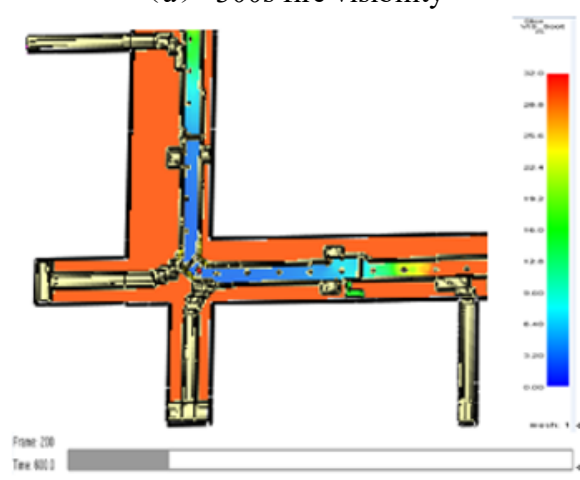

(b) 600 s fire visibility

Fig.6. Curvature 1/10 UTLT Visibility near fire

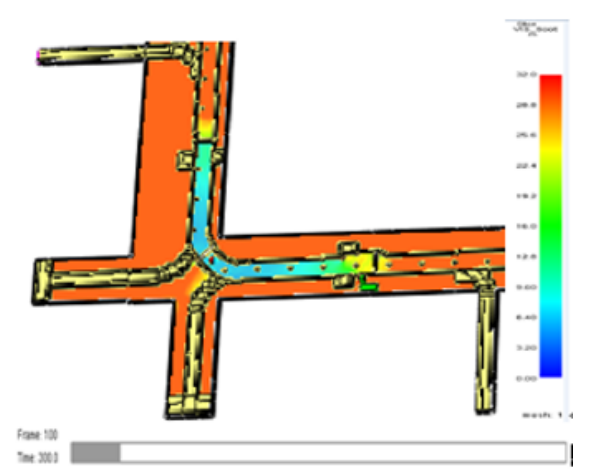

(a) 300 s fire visibility

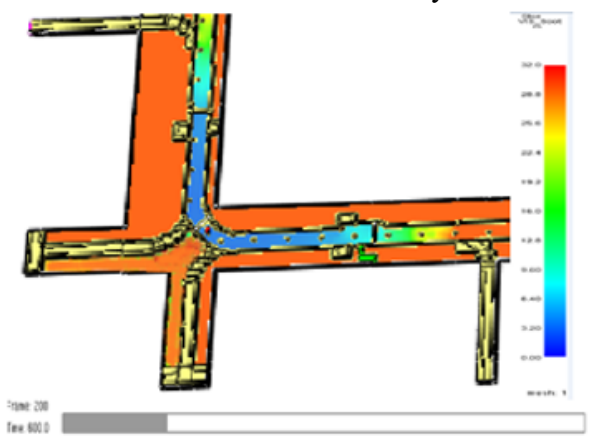

(b) 600 s fire visibility

Fig.7. Curvature 1/20 UTLT Visibility near fire

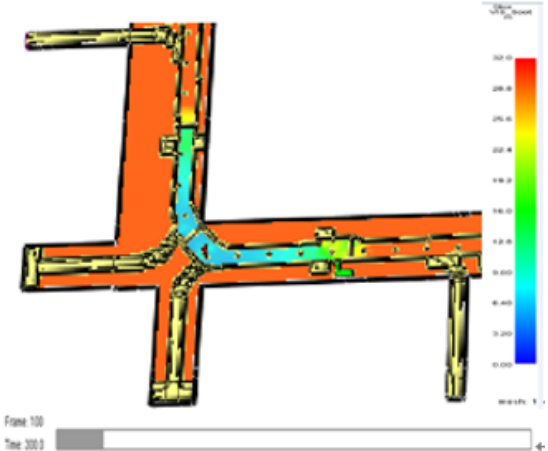

(a) 300s fire visibility

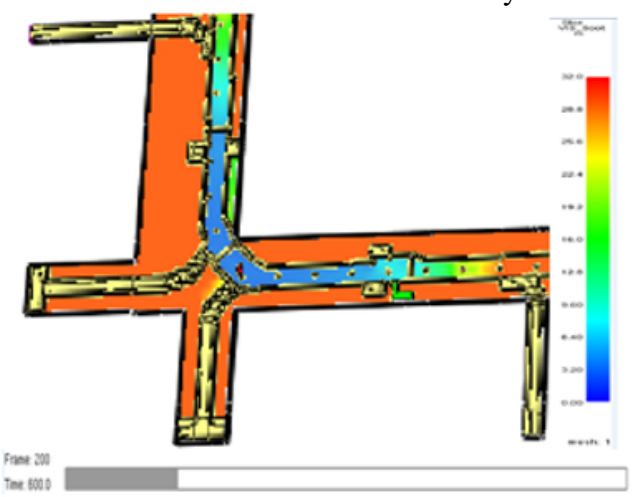

(b) 600 s fire visibility

Fig.8. Curvature 1/30 UTLT Visibility near fire

\subsection{Multiple regression prediction model establishment and analysis}

Because of the special structure and location of smallradius UTLT tunnels, there are many factors that affect the visibility of the fire in the tunnel.

This article only focuses on the multi-regression analysis of the influence of small-radius UTLT curvature on the 
fire environment visibility and explores its prediction model: $Y=\beta_{0}+\beta_{1} \chi_{1}+\beta_{2} \chi_{2}+\cdots+\beta_{\mathrm{n}} \chi_{n}+\varepsilon$.
The details of the model factors, see Table 1:

Table 1. Model factors of small radius UTLT curvature impact on fire visibility

\begin{tabular}{|c|c|c|c|}
\hline & Variable name & $\begin{array}{l}\text { Numeric } \\
\text { type }\end{array}$ & Factor description \\
\hline \multirow[t]{4}{*}{$\begin{array}{l}\text { Independent } \\
\text { variable } \mathrm{X}\end{array}$} & Curvature $\chi_{1}$ & Continuous & $\begin{array}{l}\text { With reference to relevant specifications, the } \\
\text { curvature of the tunnel is } 1 / 10,1 / 20 \text {, and } 1 / 30 \text {. }\end{array}$ \\
\hline & Time $(s) \chi_{2}$ & Continuous & This article studies the time of fire field is $1800 \mathrm{~S}$. \\
\hline & $\begin{array}{l}\text { Fire source location } \\
\qquad(\mathrm{m}) \chi_{3}\end{array}$ & Continuous & $\begin{array}{l}\text { Study the location of the fire source and } 30 \mathrm{~m} \text { from } \\
\text { the wind direction up and down the fire source }\end{array}$ \\
\hline & wind direction $\chi_{4}$ & Discrete & $\begin{array}{l}\text { Upwind and downwind are assigned } 1,2 \text { respectively, } \\
\text { and the fire source is assigned } 0\end{array}$ \\
\hline $\begin{array}{l}\text { Dependent } \\
\text { variable Y }\end{array}$ & visibility $(\mathrm{m}) \mathrm{Y}$ & Continuous & $\begin{array}{l}\text { The visibility of the fire field is affected by many } \\
\text { factors. This article only discusses the prediction } \\
\text { model of curvature near the fire source. }\end{array}$ \\
\hline
\end{tabular}

The Pyrosim simulation data was analyzed using statistical SPSS software. The results are shown in Table 2. According to Table 2, the available coefficient R2 is 0.542 , and the standard error is 5.50597 . It can be seen that there is a linear correlation between visibility and radius of curvature $\chi_{1}$, time $\chi_{2}$, distance $\chi_{3}$ to the

location of the fire source, and wind $\chi_{4}$ direction near the small radius UTLT fire source.

Table 2.Overview of regression models

\begin{tabular}{|c|c|c|c|c|}
\hline Model & $\mathrm{R}$ & R Square & Adjusted R Square & Error of the Estimate \\
\hline 1 & .736 & .542 & .538 & 5.50597 \\
\hline
\end{tabular}

The coefficients of each variable obtained by regression model coefficient table 3 are:

$$
\begin{aligned}
& \beta_{0}=19.742 \quad \beta_{1}=-11.589, \quad \beta_{2}=-0.011, \\
& \beta_{3}=-0.013, \beta_{4}=0.237 .
\end{aligned}
$$

Therefore, the regression mathematical model for the temperature around the fire source curvature is:

$Y=-11.589 \chi_{1}-0.011 \chi_{2}-0.013 \chi_{3}+0.237 \chi_{4}+19.742$

\begin{tabular}{|c|c|c|c|c|c|c|}
\hline & \multirow[t]{2}{*}{ Model } & \multicolumn{2}{|c|}{ Unstandardized Coefficients } & \multirow{2}{*}{$\begin{array}{c}\begin{array}{c}\text { Standardized } \\
\text { Coefficients }\end{array} \\
\text { Beta }\end{array}$} & \multirow{2}{*}{ Sig. } & \multirow{2}{*}{$\mathrm{t}$} \\
\hline & & $\mathrm{B}$ & Std. Error & & & \\
\hline \multirow{5}{*}{1} & (Constant) & 19.742 & 0.745 & & 0.000 & 26.491 \\
\hline & $\mathrm{X} 1$ & -11.589 & 7.982 & -0.042 & 0.147 & -1.452 \\
\hline & $\mathrm{X} 2$ & -0.011 & 0.000 & -0.735 & 0.000 & -25.316 \\
\hline & $\mathrm{X} 3$ & -0.013 & 0.033 & -0.023 & 0.696 & -0.392 \\
\hline & $\mathrm{X} 4$ & 0.237 & 0.576 & 0.024 & 0.681 & -0.412 \\
\hline
\end{tabular}

Table 3. Regression coefficients

\subsection{Predictive model test}

Through calculations, we can see from Table 4 that the F statistic is 160.792 , and the critical value for which the degree of freedom ( $\mathrm{p}, \mathrm{n}-\mathrm{p}-1)$ can be determined by examining the F distribution table is $\mathrm{F} 0.05(4,544)=2.37$.
Obviously, the $\mathrm{F}$ is much larger than the critical value, so reject $\mathrm{H} 0$, the regression equation is significant, that is, all the independent variables have a significant influence on $\mathrm{Y}$.

Table 4. Test of regression model

\begin{tabular}{ccccccccc}
\hline Model & R & RSquare & $\begin{array}{c}\text { Adjusted R } \\
\text { Square }\end{array}$ & $\begin{array}{c}\text { Std. Error of } \\
\text { the Estimate }\end{array}$ & F & df1 & df2 & Sig. F \\
\hline 1 & .736 & .542 & .538 & 5.50597 & 160.792 & 4 & 544 & .000 \\
\hline
\end{tabular}

Figure 9 shows the residual P-P graph, where the slashes represent a normal distribution with a mean of zero. It can be seen from the figure that the scattered points are densely distributed near the diagonal line, indicating that the random variable residual obeys the normal distribution, that is, the selected sample also obeys the normal distribution, which further illustrates the reliability of the model. 


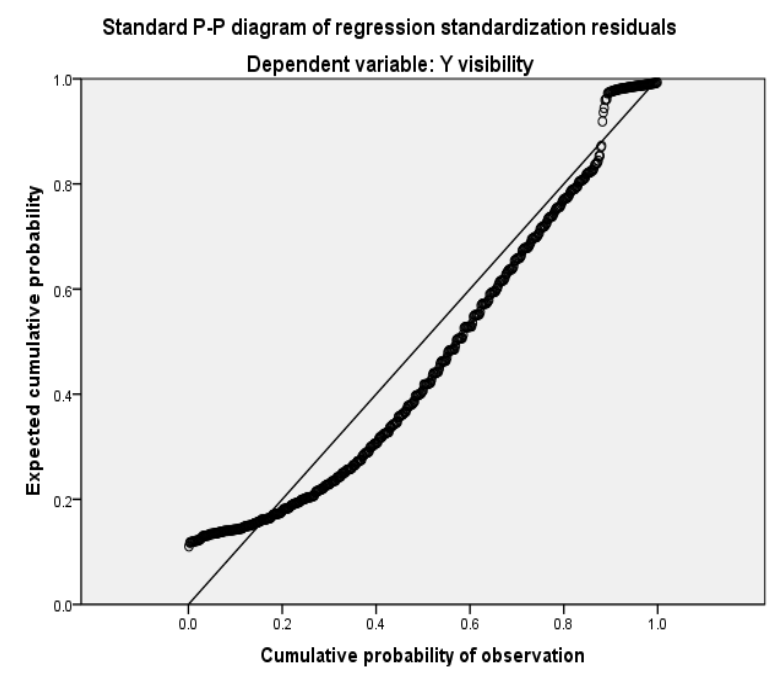

Fig.9. Residual P-P diagram

\section{Conclusions}

This study is based on fire dynamics simulation software Pyrosim and SPSS software modeling of small radius UTLT, then analysis of the impact of curvature on the fire environment visibility of the regression model. The results are:

(1) The small-radius UTLT tunnel is a typical narrow and confined space, and the structure is complex and the fire situation is severe. Due to the existence of curvature in the tunnel, it is easy to form the phenomenon of "smoke turning", which greatly reduces the visibility of the fire field and seriously threatens people's rapid escape.

(2) With the increase of curvature, the easier the accumulation of smoke in UTLT, the lowest visibility of the fire field can reach $4 \mathrm{~m}$ far below the safety limit. Curvature is one of the important factors that affect the visibility of the UTLT near the small radius of fire.

(3) The multiple-regression analysis shows that the curvature of UTLT with small radius is negatively correlated with the visibility near the fire source. The established regression model has certain reliability, and can provide theoretical guidance and reference for the smoke prevention, curvature design, and personnel safety evacuation of UTLT small radius loops.

The environmental visibility of small-radius UTLT fire is affected by many factors. This paper focuses on the influence of UTLT curvature on the visibility near the fire source, and establishes a mathematical model. In the future, the influence of multi-factor coupling will be further studied.

\section{References}

1. Dong xing guo. Numerical simulation of smoke exhaust effect in urban traffic link tunnel with longitudinal ventilation[D].BeiJing University of Technology, 2014.

2. Li Si Cheng. Study on fire smoke flow characteristics and optimal control strategies in urban traffic tunnel.BeiJing University of Technology, 2016.

3. Chen qing.Numerical simulation of water mist suppression of Urban Traffic Link Tunnel fires[D]. Xi hua University,2017.

4. He Jia.Numerical Simulation of Ventilation Accident Ventilation in Small Radius Curve Tunnel[D].Central South University, 2008.

5. LI Tao. Small-scale experimental study on characteristics of spiral tunnel fire $[\mathrm{J}]$. Journal of Safety Science and Technology2017, 13(3): 137-143

6. Zhou Xi.Study on the safety technical of urban underground traffic space[D].Beijing University of Civil Engineering and Architecture, 2015.

7. Hao Aizhi.Experimental Study on the Distribution of Carbon Monoxide Concentration and Temperature rise in Tunnel Fires[J]. Chinese Journal of Underground Space and Engineering2016, 12(5): 1185-1191

8. LIU Yi-xuan.Experimental study of the influence ofthe longitudinal ventilation on the fire parameters in highway tunnels $[\mathrm{J}]$. Journal of Safety and Environment2017,17( 5) : 1777-1781.

9. WU Can, HE Jia, NI Tianxiao. R esearch on the smoke movement insmall radius curvilinear tunnel fires $[\mathrm{J}]$. Fire Science and Technology, 2014, 33( 1$)$ : 37-40.-

10. THOMAS P H. The movement of buoyant fluid against astream and the venting of underground fires[R]. BorehamWood, Herts: Fire $R$ esearch Station, 1958.

11. Kunikane Y., Kawabata N., Yamada T., et al. Influence of stationary vehicles on backlayering characteristics of fire plume in a large cross section tunnel[J]. JSME International Journal Series B, 2006, 49(3): 594-600.

12. HU L H. Studies on thermal physics of smoke movement in tunnel fires [D] . Hefei: State Key Laboratory of FireScience University of Science and Technology of China, 2006. (in Chinese)

13. Huang jian bo.Analysis on influence factor of smoke visibility in subway tunnel fire based on the orthogonal experiment[J]. Fire Science and Technology, 2017, 36(11): 1521-1524.

14. Xu jin tao. Simulation and analysis of the impact of different soot-yields to the visibility in the underground carpark[J]. Journal of Engineering Thermophysics, 2014, 1(35): 171-175.

15. Li Xiao Ju.The study of fire smoke motion mechanism about urban underground link tunnel under different curvature and ventilation condition [D].Sichuan Normal University,2016.

16. LONG Xin-feng, ZHANG Xue-qin. Analysis of the dormitory building fire risk based on the Pyrosim simulation [J].Journal of Safety and Environment,2017, 17(4): 1348-1353. 
17. WANG Feng, DONG Guohai, WANG Mingnian. On the critical airvelocity for fire smoke control in a curved tunnel $[\mathrm{J}]$. Modern Tun-neling Technology, 2015( 5) : 84-89.

18. Ji jie.Experimental study on the entrainment mass flow rate across the smoke layer interface during horizontal spread in a long channel $[\mathrm{J}]$. Journal of China University of Science and Technology.2009,39(7) : 738-743.

19. Liu Ai Yu.Spss basic analysis tutorial[M]. Peking University Press, 2014. 